\title{
The flexor carpi radialis H-reflex in polyneuropathy: relations to conduction velocities of the median nerve and the soleus H-reflex latency
}

\author{
R J SCHIMSHEIMER,* B W ONGERBOER DE VISSER, *† B KEMP, $\ddagger$ L J BOUR $†$ \\ From the Departments of Clinical Neurophysiology and Neurology, Academic Medical Centre, $\uparrow$ and St Lucas \\ Hospital,* Amsterdam, and Academic Hospital, $\ddagger$ Leiden, The Netherlands
}

SUMMARY In 80 controls latencies of flexor carpi radialis (FCR) and in 94 controls latencies of soleus $\mathrm{H}$-reflexes correlated well with length of the extremity, body height and age. Multiple regression equations using latency as a variable dependent on age and body height can be best used in practice when both reflexes are employed for demonstration of proximal pathology. The majority $(69 \%)$ of 93 patients with various polyneuropathies showed abnormalities in both reflexes illustrating that proximal nerve segments are frequently involved. Four per cent had abnormal FCR Hreflexes with normal soleus $\mathrm{H}$-reflexes whereas the reverse was found in $19 \%$ of the patients. Abnormal FCR H-reflexes occurred with normal motor and sensory conduction velocities in the peripheral part of the median nerve in $14 \%$, whereas the reverse was seen in $12 \%$, indicating that FCR H-reflex examination is a valuable supplement to conventional conduction studies for detection of electrophysiologically existing pathology.

Recording of the H-reflex of the flexor carpi radialis (FCR) muscle has proved to be an effective tool for the examination of damage of the proximal segment of the median nerve. ${ }^{12}$ It has been demonstrated that performing studies of the H-reflex of the soleus muscle is useful in assessing polyneuropathy, ${ }^{3-5}$ but data on the FCR H-reflex in this type of nerve dysfunction are not yet available.

The object of this study was to determine the relationship between sensory (SNCV) and motor nerve conduction velocity (MNCV) in the elbowwrist segment of the median nerve, that is, the distal segment, and FCR H-reflex latencies elicited from the same nerve in a group of patients with well established polyneuropathy. In addition, changes of the FCR H-reflex were compared with those of the soleus H-reflex. Furthermore, in a group of control subjects, it has been evaluated whether both reflexes show similar relationships to the length of the extremity tested or to body length only, which would be easier to measure for practical purposes.

Address for reprint requests: BW Ongerboer de Visser, MD, Department of Clinical Neurophysiology (D2), Academic Medical Centre, Meibergdreef 9, 1105 AZ-Amsterdam, The Netherlands.

Received 3 April 1986.

Accepted 19 June 1986

\section{Methods and materials}

The electrophysiological examinations were performed in an airconditioned room with the temperatures controlled between $23^{\circ} \mathrm{C}$ and $25^{\circ} \mathrm{C}$. Surface temperatures of the lower arm ranged from $32^{\circ}$ to $34^{\circ} \mathrm{C}$ and of the dorsum of the foot from $30^{\circ}$ to $32^{\circ} \mathrm{C}$.

The method used to elicit the H-reflex of the FCR muscle has been described previously. ${ }^{1}$ The median nerve in the cubital fossa was stimulated by a bipolar surface electrode with the cathode placed proximally and reflex responses were recorded by a needle electrode. The stimulus frequency was one per 5 seconds, and the pulse duration $0.5 \mathrm{~ms}$. The voltage was increased and multiple recordings were made. The potential with the shortest latency time to the start of first deflection from the baseline was measured.

For determination of the soleus $\mathrm{H}$-reflex one pair of recording electrodes were placed longitudinally about $3 \mathrm{~cm}$ apart over the midline of the soleus muscle, the active electrode being about $2 \mathrm{~cm}$ below the insertion of the gastrocnemius on the Achilles tendon and the reference electrode further distally. The tibial nerve in the popliteal fossa was stimulated by a bipolar surface electrode with the cathode placed proximally. The stimuli were square pulses of $1 \mathrm{~ms}$ and were applied at 2 second intervals. With the stimulating electrodes in the right position the voltage was increased and subsequent $\mathrm{H}$-reflexes were recorded. The reflex with the shortest latency time to the start of the first deflection from the baseline was measured.

In 80 control subjects, between the ages 14 and 83 years 
Table 1 Types of neuropathy, EMG types of neuropathy, motor (MNCV) and sensory (SNCV) nerve conduction velocities in the median nerve, the number of absent sensory nerve $(S N)$ potentials in the median nerve, and changes of the flexor carpi radialis (FCR) and soleus $H$-reflex latencies (lt) in the 93 patients with polyneuropathy

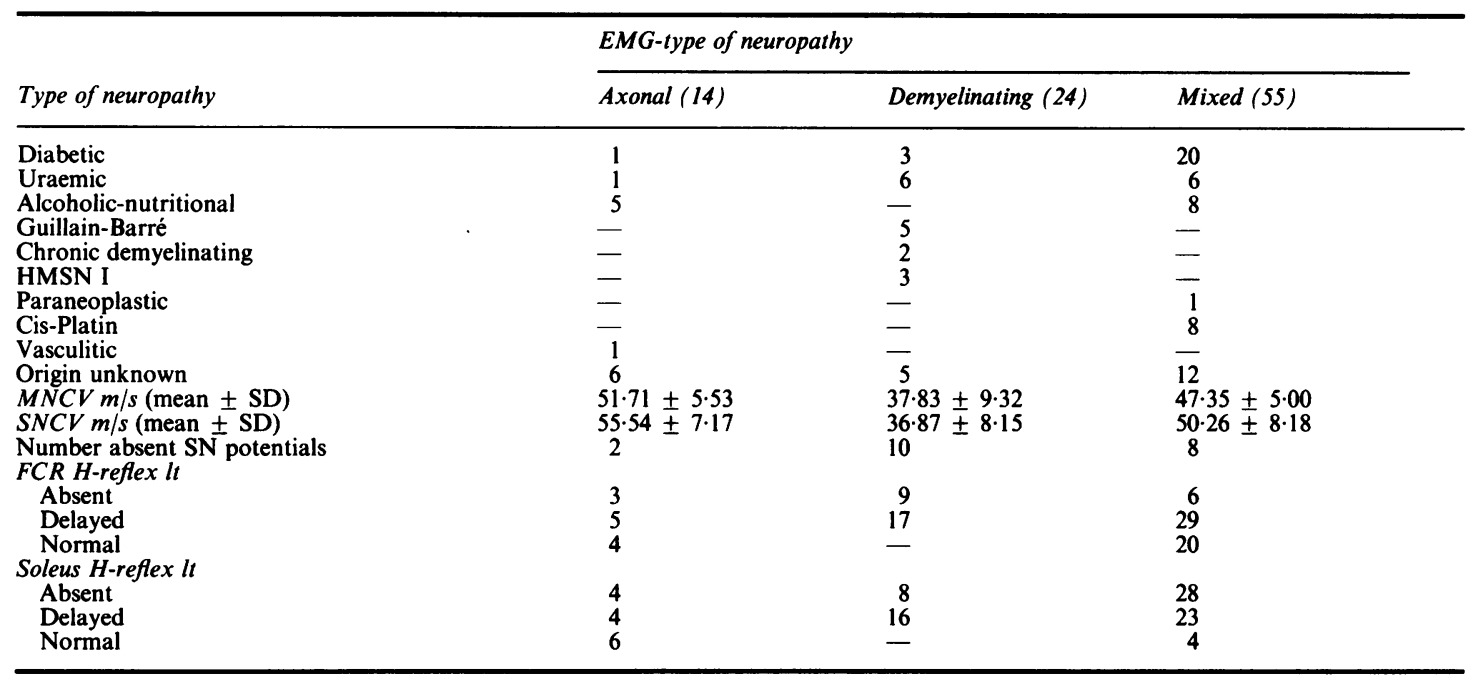

(mean 49.6 years), FCR H-reflexes and in 94 control subjects, between the ages 19 and 86 years (mean 40.7 years), soleus H-reflexes were studied. Both reflex studies were performed in most of the subjects of the two groups. Age, arm length, leg length and body length were recorded. Arm length was measured from the top of digit 3 to the C6 spine with the arm pronated on the coronal plane and abducted at a right angle. Leg length was measured from the anterior iliac crest to the lateral side of the heel.

The patient group consisted of 93 patients, between ages 26 and 84 years (mean 62.7 years), with clinical and laboratory evidence of various neuropathies (table 1). All patients had conventional motor nerve conduction studies of both peroneal and median nerves, and EMG studies with concentric needle electrodes. In all of them the antidromic SNCV of the median nerve from elbow to wrist was measured by recording potentials from the digital nerves at the base of the index finger by means of ring electrodes. Most patients also had antidromic SNCV studies of one sural nerve with use of stimulating surface electrodes over the nerve at the lower edge of the lateral belly of the gastrocnemius and recording surface electrodes over the lateral ankle. Age, arm length and body height of each patient were recorded.

\section{Results}

\section{Controls}

Both the FCR and the soleus H-reflex could be elicited in all control subjects. The mean latency of the FCR H-reflex was $16.84 \mathrm{~ms} \pm 1.33$ standard deviation (SD) with a right-left difference of $0.002 \mathrm{~ms} \pm$ $0.42 \mathrm{SD}$ and the mean latency of the soleus $\mathrm{H}$-reflex was $29.97 \mathrm{~ms} \pm 2 \cdot 10 \mathrm{SD}$ with a right-left difference of $0.09 \mathrm{~ms} \pm 0.70 \mathrm{SD}$. There was no relationship to gender.
Single regression equations were calculated for FCR and soleus $\mathrm{H}$-reflex latency times (ms) as a function of arm, leg or body length $(\mathrm{cm})$ and age $(\mathrm{yr})$ respectively; FCR H-reflex latency $=3.54+0.1526$ $x$ arm length \pm 0.97 (SD), FCR H-reflex latency $=$ $3.07+0.0811 \times$ body length \pm 1.01 (SD) (fig la), and FCR H-reflex latency $=15.82+0.0206 \times$ age 1.28 (SD) (fig la) and soleus H-reflex latency $=16.09$ $+0.1346 \times$ leg length $\pm 1.79(\mathrm{SD})$, soleus H-reflex latency $=11.84+0.1056 \times$ body length \pm 1.80 (SD) (fig $1 \mathrm{~b}$ ) and soleus H-reflex latency $=28 \cdot 55+$ $0.0349 \times$ age \pm 2.02 (SD) (fig lb). These relationships were highly significant (Student's two tailed $t$ test: $\mathrm{p}<0.001$ ).

Multiple regression equations using latency time (ms) as a variable dependent on age (yr) and limb or body length $(\mathrm{cm})$ were calculated: FCR H-reflex latency $=1.29+0.1630 \times$ arm length $+0.0270 \times$ age $\pm 0.83(\mathrm{SD})$ and FCR H-reflex latency $=-0.44$ $+0.0925 \times$ body length $+0.0316 \times$ age \pm 0.83 (SD), and soleus H-reflex latency $=6.75+0.1964 \times$ leg length $+0.0731 \times$ age $\pm 1.38(\mathrm{SD})$ and soleus H-reflex latency $=3.00+0.1419 \times$ body length + $0.0643 \times$ age $\pm 1.47(\mathrm{SD})$. All dependencies were highly significant $(\mathrm{p} \ll 0.001)$.

The SDs indicate that the lengths of limbs or body heights have about the same predictive value for the latency times of both reflexes. For the FCR H-reflex latency the SD decreased by $25 \%$ using the single regression on body length and by a further $18 \%$ using the multiple regression equation. For the soleus Hreflex latency the SD decrease amounted to $14 \%$ and 

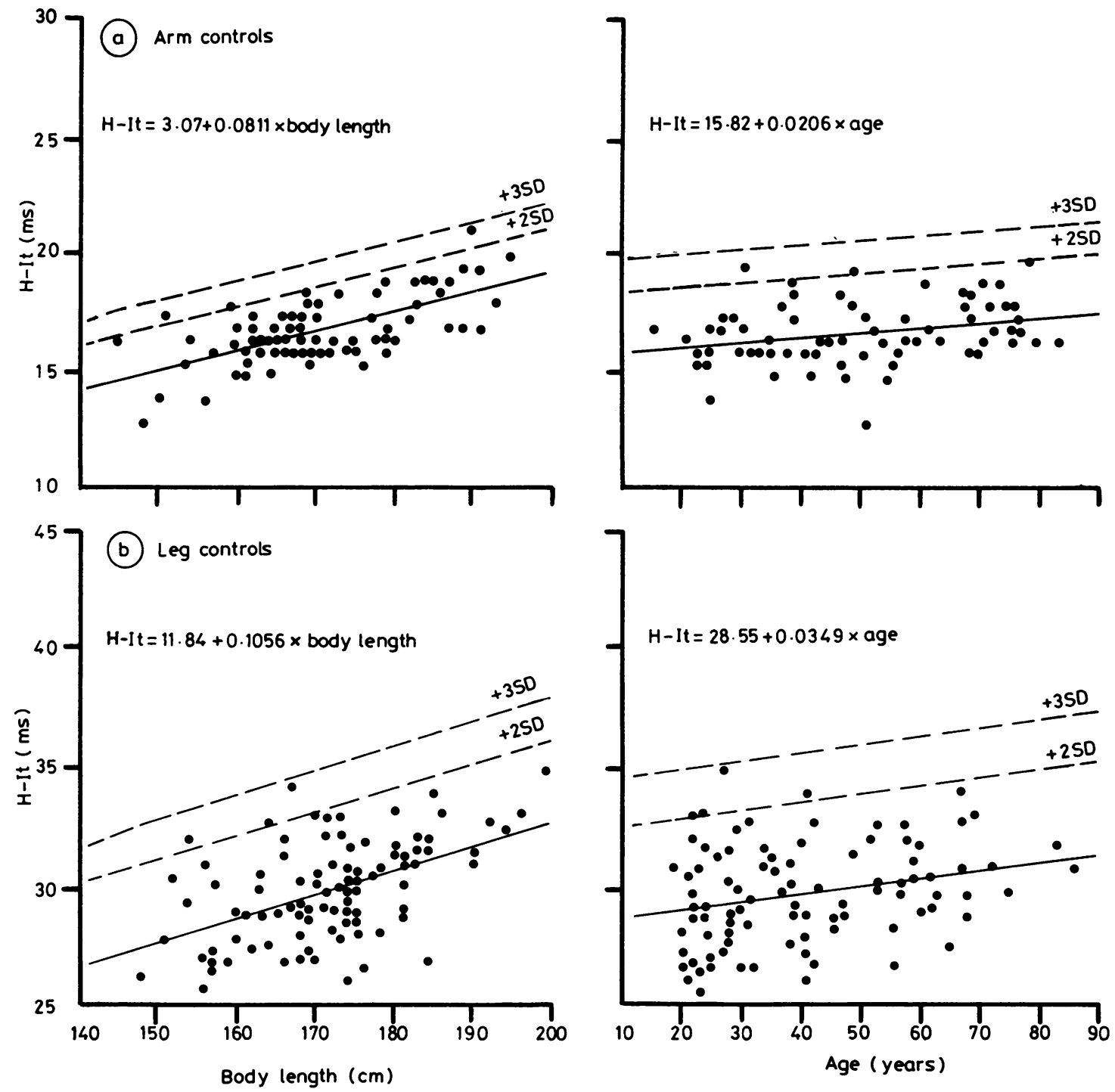

Fig 1 (a) Relationships of body length and age of control subjects to H-reflex latency time (H-lt) of the flexor carpi radialis $H$-reflex. (b) Relationships of body length and age of control subjects to H-lt of the soleus H-reflex. In this study upper crossing of the $+3 S D$ level is considered abnormal.

$18 \%$, respectively. Upper crossing of the +3 SD level after regression on both body height and age was considered abnormal.

\section{Patients}

Our 93 polyneuropathy patients could be divided into three groups according to the electrophysiological criteria reported by Shahani $e t \mathrm{al}^{6}$ and based on conduction velocities in the peroneal and sural nerves as well as EMG findings in muscles of the lower extremities (table 1). Group 1 consisted of 14 patients with axonal disorders, group 2 consisted of 24 patients with demyelinating neuropathies and group 3 consisted of 55 patients with mixed axonal and demyelinating disorders.

With respect to the elbow-wrist segment of the median nerve the mean value of the MNCV in the group with axonal disorders was $51.71 \mathrm{~ms} \pm 5.53 \mathrm{SD}$ 
Table 2 Combinations of normal and abnormal findings in the flexor carpi radialis and soleus $H$-reflex seen in the 93 patients with polyneuropathy

\begin{tabular}{|c|c|c|c|c|}
\hline & \multicolumn{4}{|c|}{ Flexor carpi radialis $\boldsymbol{H}$-reflex latency } \\
\hline & \multicolumn{3}{|c|}{ Soleus H-reflex latency } & Total \\
\hline Normal & 6 & 3 & 1 & 10 \\
\hline Delayed & 12 & 26 & 5 & 43 \\
\hline Absent & 6 & 22 & 12 & 40 \\
\hline Total & 24 & 51 & 18 & 93 \\
\hline
\end{tabular}

in the group with demyelinating neuropathies $37.83 \mathrm{~ms} \pm 9.32 \mathrm{SD}$, and in the group with mixed disorders $47.35 \mathrm{~ms} \pm 5.00 \mathrm{SD}$ (table 1 ). The mean value in the mixed neuropathies was similar (Student's $t$ test: $\mathrm{p}<0.07)$ to the one in the axonal disorders. Both were markedly $(p<0.01)$ faster than the mean value in the demyelinating neuropathies. With respect to the SNCV (table 1) the mean value in the group with axonopathies was $55.54 \mathrm{~ms} \pm 7 \cdot 17 \mathrm{SD}$, in the group with demyelinating neuropathies $36.87 \mathrm{~ms} \pm$ $8.15 \mathrm{SD}$ and in the group with mixed disorders $50.26 \mathrm{~ms} \pm 8 \cdot 18 \mathrm{SD}$. The mean values were similar $(\mathrm{p}$ $<0 \cdot 1)$ for axonal and mixed neuropathies. The above findings are in contrast to the demyelinating neuropathies, which had a significant $(p<0.001)$ slower conduction velocity than found in both the axonopathies and the mixed neuropathies. The formulae of the normal MNCV and of the normal SNCV of the distal segment of the median nerve used in our laboratories are $61.51-0.1020 \times$ age $(\mathrm{yr})+4.00 \mathrm{SD}$ and $65 \cdot 71-0.0760 \times$ age $(\mathrm{yr})+4 \cdot 76 \mathrm{SD}$, respectively. Using the -3 SD level in the formulae as lower limit of normal the MNCV has been decreased in 24 whereas being normal in the remaining 69 patients. The SNCV has been decreased in 30 whereas remaining normal in 43 patients. In 20 patients no sensory potentials could be elicited in the median nerves; two $(14 \%)$ belonged to the group of 14 patients with axonopathies, eight $(14 \%)$ to the group of 55 patients with mixed neuropathies and $10(42 \%)$ to the group of 24 patients with demyelinating neuropathies. Considering delayed SNCVs and absent sensory median nerve potentials together the following combinations of MNCV and SNCV findings were seen. Both MNCV and SNCV were normal in 30 and both abnormal in 18 patients. Thirty two had abnormal SNCVs and normal MNCVs. In 13 patients MNCV was abnormal and SNCV normal.

With respect to absent $\mathrm{H}$-reflexes this was noted in 18 patients for the FCR H-reflex and in 40 patients for the soleus H-reflex (tables 1 and 2). FCR Hreflexes were delayed in 51 and soleus $\mathrm{H}$-reflexes delayed in $\mathbf{4 3}$ patients. The FCR H-reflex was normal in
24 and the soleus $\mathrm{H}$-reflex normal in 10 patients. The following combinations of reflex findings were seen (table 2). In four patients the FCR H-reflex was abnormal, absent in one patient and delayed in three patients, whereas the soleus $\mathrm{H}$-reflex was normal. In 18 patients the soleus $\mathrm{H}$-reflex was abnormal, absent in six and delayed in 12 patients, whereas the FCR $\mathrm{H}$-reflex was normal. Both FCR and soleus $\mathrm{H}$-reflexes were abnormal in 65 patients, absent in 12 patients, delayed in 26 patients, the FCR H-reflex delayed and the soleus H-reflex absent in 22 patients and the soleus H-reflex delayed and the FCR H-reflex absent in five patients. It should be noted that all 24 patients with demyelinating polyneuropathy had disturbed FCR H-reflexes in addition to an abnormal soleus $\mathrm{H}$-reflex. Both reflexes were normal in six patients.

With respect to the relationships between FCR Hreflex latency and both MNCV and SNCV in the elbow-wrist segment of the median nerve the following has been observed. In 13 patients (14\%) FCR Hreflexes were abnormal, one absent and 12 delayed, whereas median nerve conduction velocities were normal in all of these patients. In 11 patients $(12 \%)$ FCR $\mathrm{H}$-reflexes were normal, whereas conduction velocities were delayed; both MNCV and SNCV in seven, and only MNCV in four patients. In 60 patients $(65 \%)$ both FCR H-reflexes and median nerve conduction velocities were abnormal. Of these patients FCR H-reflexes were absent in 18 and delayed in 42 patients, whereas of all conduction velocities measured the MNCV only was abnormal in four, SNCV only was abnormal in six patients, and both were abnormal in the remaining patients. In nine patients (9\%) both FCR H-reflex and conduction velocity were normal. It should be noted that in these patients reflexes showed abnormal dispersed responses (fig 2a) in one patient, polyphasic responses (fig $2 b$ ) in three patients, and asynchronous small responses (fig $2 \mathrm{c}$ ) in one patient, although latencies fell into the normal range. The other patients had reflex responses with normal configurations. The three abnormal features mentioned above also occurred occasionally in patients with prolonged FCR H-reflex latencies.

Of the 75 patients with obtainable FCR H-reflexes 43 patients $(51 \%)$ showed increased latencies with +3 SD as upper limit of normal when only arm length or body height, and $36(48 \%)$ when only age was used to predict FCR H-reflex latencies. This was true for the soleus $\mathrm{H}$-reflex, obtainable in 53 patients, in $38(72 \%)$ when only body height, and in 29 patients $(55 \%)$ when only age was used to predict latencies. The multiple regression equation using latency as variable dependent on age and body height yielded abnormal FCR H-reflex latencies in 47 patients $(63 \%)$ and abnormal soleus $\mathrm{H}$-reflex latencies in 34 $(64 \%)$ patients. 

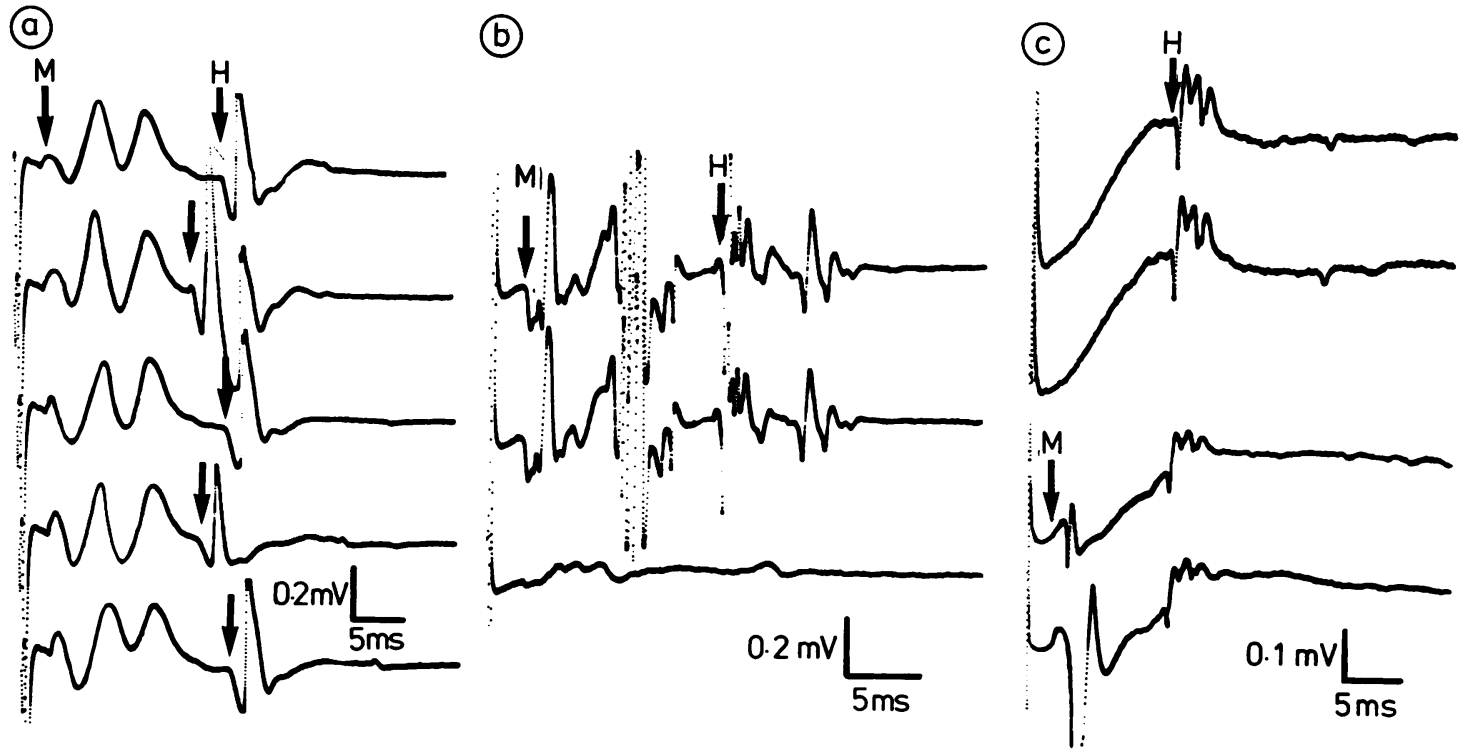

Fig 2 (a) Abnormal dispersed, (b) polyphasic and (c) asynchronous small flexor carpi radialis (FCR) H-reflex responses occurring in three patients with normal latency times of FCR H-reflexes and normal median nerve conduction velocities.

\section{Discussion}

To our knowledge conduction velocity in the most proximal segment of the median nerve by EMG means has only been evaluated by MNCV determination in the Erb-axilla nerve segment ${ }^{7}$ and by the F-wave technique ${ }^{8}$ by which functional values of motor fibres can also be studied. However, evaluation of peripheral neuropathy should include determination of SNCV as well. Simultaneous measurements of FCR H-reflex latency and the conventional MNCV and SNCV in the median nerve segment of the lower arm allow comparison of propagation velocities in proximal and distal parts of the median nerve.

In our control groups regression equations demonstrated that latencies of FCR and soleus H-reflexes can be predicted from limb length but equally accurately from body height (fig la and b). Therefore, body height alone can be used in practice when both reflexes have to be investigated for demonstration of proximal pathology. Multiple regression analyses demonstrated that prediction by age further improves accuracy for FCR and soleus H-reflexes.

The greatest percentage $(69 \%)$ of our patients showed delayed latencies or absence of both the FCR and the soleus $\mathrm{H}$-reflexes, which illustrates that proximal parts of nerves are frequently involved in neuropathy. An abnormal soleus $\mathrm{H}$-reflex with a normal FCR H-reflex was found in $19 \%$. Notable was the finding that few patients $(4 \%)$ showed an abnormal FCR H-reflex and a normal soleus H-reflex.
Considering the median nerve alone, abnormal FCR H-reflexes occurred with normal MNCV and SNCV in $14 \%$ of the patients. In these cases pathology of the proximal median nerve segment could be overlooked when nerve conduction in the elbow-wrist segment only was performed. Nerve conduction studies in the peripheral part were abnormal with normal FCR H-reflex latencies in $12 \%$ of the patients. This and the reversed findings in the former patients demonstrate that either the proximal or the distal part of the median nerve was the predominant location of nerve damage and therefore both nerve conduction and reflex examinations should be performed to show electrophysiologically existing pathology.

FCR H-reflexes could not be elicited in 18 patients $(19 \%)$ and soleus $\mathrm{H}$-reflexes were unelicitable in 40 patients $(43 \%)$. Most of these patients belonged to the group with demyelinating neuropathies and, as expected, showed the slowest conduction velocities and most frequently absent sensory potentials also. Only few data are available on reoccurrence of absent H-reflexes during recovery of neuropathy. ${ }^{9}$ Probably, this may happen in only a small percentage after a considerably long time period. Therefore, it is of interest that FCR H-reflexes could often be recorded when soleus $\mathrm{H}$-reflexes were unelicitable. This may be of importance for follow-up studies to evaluate either worsening or improving of proximal neuropathy.

Nine patients $(9 \%)$ had normal median nerve conduction velocities and FCR H-reflexes with normal latencies and were therefore classified as subjects with 
normal FCR H-reflexes. Five of them, however, showed reflexes with clearly abnormal appearances (fig 2a-c). These pattern changes are on the one hand difficult to quantify with usual methods, but on the other hand it might also be reasonable to consider them abnormal. Three patients had polyphasic responses (fig $2 \mathrm{~b}$ ), suggesting collateral reinnervation, another had markedly decreased responses (fig $2 \mathrm{c}$ ), suggesting that axonal degeneration took place, while a fifth patient showed successive responses with different latencies and wave forms (fig 2a). In the latter it was possible to identify separate motor unit responses, appearing either alone or in combination, relying on their characteristic wave form and latency time. The abnormal dispersion of responses, probably due to desynchronisation of impulses, would provide evidence of a focal lesion of the proximal part of the median nerve. These observations further demonstrate that the usual triphasic appearance of the $\mathrm{H}$ reflex may be replaced by a disturbed one earlier on in the course of the disease than prolonged latency time occurs, even at a time when the direct muscle response has a normal shape, which was seen in one patient (fig 2c). The finding supplements those of Lachman et $a l^{4}$ who reported that prolongations of latencies may occur earlier on in the course of polyneuropathy than asynchrony of $\mathrm{H}$-reflex responses.

With respect to the significant age influence on $\mathrm{H}$ reflexes, it could be expected that, with multiple regression equations using latency as a variable dependent on age and body height, more abnormalities of soleus $\mathrm{H}$-reflexes would be found in the patient group than when using single regression equation for latency as function of body height. However, of the 53 obtainable soleus $\mathrm{H}$-reflexes single regression analysis yielded 38 and multiple regression analysis only 34 soleus H-reflexes with prolonged latencies. Soleus $\mathrm{H}$-reflexes in one patient, aged 48 years, were considered to be normal and in five patients, all older than 63 years, to be abnormal when using single regression analysis only.

Apparently, the five abnormal recordings obtained by single regression analysis do not take into account the normal prolongation of the latencies in these patients caused by their advancing age. By the same token the one normal recording is, probably, also unreliable. The above illustrates that regression correction using both body height and age is more valuable for detection of abnormal latencies.

In conclusion, the present study has shown that recording of the FCR H-reflex is a valuable supplement to conventional conduction studies for detection of pathology of the median nerve in patients with polyneuropathy.

The authors appreciate the help of Dr DN Velis in reading the English text.

\section{References}

1 Ongerboer de Visser BW, Schimsheimer RJ, Hart AAM. The H-reflex of the flexor carpi radialis muscle: a study in controls and radiation induced brachial plexus lesions. J Neurol Neurosurg Psychiatry 1984;47: 1098-101.

2 Schimsheimer RJ, Ongerboer de Visser BW, Kemp B. The flexor carpi radialis $\mathrm{H}$-reflex in lesions of the sixth and seventh cervical nerve roots. $J$ Neurol Neurosurg Psychiatry 1985;48:445-9.

3 Guiheneuc P, Bathien N. Two patterns of results in polyneuropathies investigated with the H-reflex. $J$ Neurol Sci 1976;30:83-94.

4 Lachman T, Shahani BT, Young RR. Late responses as aids to diagnosis in peripheral neuropathy. $J$ Neurol Neurosurg Psychiatry 1980;43:156-62.

5 Troni W, Carta Q, Cantello R, Caselle MT, Rainero I. Peripheral nerve function and metabolic control in diabetes mellitus. Ann Neurol 1984;16:178-83.

6 Shahani BT, Halperin JJ, Boulu P, Cohen J. Sympathetic skin response-a method of assessing unmyelinated axon dysfunction in peripheral neuropathies. $J$ Neurol Neurosurg Psychiatry 1984;47:536-42.

7 Ginzburg M, Lee M, Ginzburg J, Alba A. Median and ulnar nerve conduction determinations in the Erb's point-axilla segment in normal subjects. $J$ Neurol Neurosurg Psychiatry 1978;41:444-8.

8 Kimura J. In: Kimura J, ed. Electrodiagnosis in Diseases of Nerve and Muscle: Principles and Practice. Philadelphia: FA Davis, 1983:353-77.

9 Ongerboer de Visser BW, Tiessens G. Polyneuropathy induced by Cisplatin. In: Clifford Rose F, Fields WS, eds. Neuro-oncology. Basel: Karger. (Prog Exp Tumor Res Vol 29), 1985:190-6. 